\title{
Cyclosporin and mitochondria: a neuroprotective approach
}

Sir,

Cyclosporin A (CsA) an immunophilin, discovered in 1969 and approved in 1983 to be used as immunosuppressant agent and is widely used in organ transplantation and auto-immune disorders. Its ability to alter mitochondria and apoptotic pathways makes it attractive agent to be employed in variety of diseases including age related neurodegenerative diseases. Mitochondria play pivotal role in cell energetics and reactive oxygen species production and are known to be key regulator of apoptosis hence it is important in a wide range of diseases. The structural and functional properties of mitochondria enable the targeting of drugs supposed to modulate the function of organelle for therapeutic advantage. By targeting mitochondria we can prevent oxidative damage associated with neurodegenerative diseases and ischemia and reperfusion tissue injury. Similarly targeting Bcl-2 can be helpful in cancer by triggering apoptosis. Uncouplers of oxidative phosphorylation can be exploited in management of obesity and diabetes. In the early years of 1990s CsA was shown to possess neuroprotective property ${ }^{1}$, this was the turning point which led to exploration of hidden neuroprotective potential of cyclosporine. Cyclosporine has shown its neuroprotective potential in various animal models of stroke and head injury. ${ }^{2-4}$ Cyclosporin A, at therapeutically relevant concentrations, acts directly on neural precursor cells to enhance their survival both in vitro and in vivo. ${ }^{5}$ Thus Cyclosporin $\mathrm{A}$ is a promising agent in the development of regenerative strategies which aim to repair and regenerate damaged or diseased Central Nervous System tissue. It has been shown to block mitochondrial permeability transition pores (MPTP) which in open state causes mitochondrial dysfunction and preservation of mitochondrial function assessed by tissue oxygen consumption, directly translated into improvements in motor and cognitive behavior. ${ }^{4}$ At higher doses the Cyclosporin $\mathrm{A}$ as well as Tacrolimus have been shown to markedly decrease expressions of Cytochrome $\mathrm{C}$, apoptosis inducing factor (AIF), caspase3 and inhibited apoptosis pathways. Above mentioned chemical substances involve mitochondria which play critical role in apoptosis, and their inhibition are supposed to be responsible for neuroprotection, so cyclosporine can be called as mitochondrial medicine. Similar mechanisms are thought to mediate the cardioprotective effects of cyclosporine and trials for exploration of this potential are being carried out. (CIRCUS TRIAL). These unconventional effects of CsA open a large window of therapeutic application. Since apoptosis is the ultimate process in many diseases, inhibition of apoptosis by CsA can be exploited in many diseases. The pathophysiology of nearly all neurodegenerative disease involve apoptosis, hence CsA is an attractive target for these diseases. Although the CsA has shown promise in preclinical studies but in the clinical settings failed to replicate the results. In one of the trial of patients with acute traumatic brain injury who received cyclosporine administered intravenously, with treatment initiated within 8 hours of injury, the rate of mortality or other adverse events was not significantly different from that of the placebo group. ${ }^{6}$ Although the results were not encouraging, still trials are in phase $2 / 3$ for its neuroprotective property in cases of moderate to severe traumatic brain injury and stroke are being carried out. $^{7}$ Currently we don't have any established neuroprotective agent because of complex pathophysiology of TBI and stroke. The lack of knowledge between temporal relationship of pathophysiologic events with drug administration remains a significant cause of failure of drugs in clinical studies along with the methodological errors. There are no standardized guidelines to carry out these sort of studies with different endpoints (short term vs long term), different time window (short vs long term duration of drug treatment) in preclinical and clinical studies respectively which are prone for failure. In clinical setting the trials are limited by inappropriate outcome measures, failure to target specific head injury and stroke subtypes, and not able to modify the ischemic penumbra. How much cyclosporine will be successful as a neuroprotective agent in cases of traumatic brain injury or stroke, it will be evident only after completion of clinical trial.

\author{
Alok Singh $^{1} *$, Vivek Khare \\ ${ }^{1}$ Department of Pharmacology, G. R. Medical College, \\ Gwalior 474001, Madhya Pradesh, India \\ ${ }^{2}$ Department of Pathology, L. N. Medical College, \\ Bhopal 462042, Madhya Pradesh, India \\ *Correspondence to: \\ Dr. Alok Singh, \\ E-mail: draloksingh410@gmail.com
}




\section{REFERENCES}

1. Uchino H, Elmér E, Uchino K, Lindvall O, Siesjo BK. Cyclosporin A dramatically ameliorates CA1 hippocampal damage following transient forebrain ischaemia in the rat. Acta Physiol Scand 1995; 155:469-71.

2. Kilbaugh TJ, Bhandare S, Lorom DH, Saraswati M, Robertson CL, Margulies SS. Cyclosporin A preserves mitochondrial function after traumatic brain injury in the immature rat and piglet. $\mathrm{J}$ Neurotrauma 2011;28:763-74.

3. Leger PL, et al. Evaluation of cyclosporine A in a stroke model in the immature rat brain. Exp Neurol 2011;230:58-66.

4. Xie Z, et al. Neuroprotective effect of Cyclosporin $\mathrm{A}$ on the development of early brain injury in a subarachnoid hemorrhage model: a pilot study. Brain Res 2012;1472:113-23.

5. Hunt $\mathbf{J}$, et al. Cylosporin A has direct effects on adult neural precursor cells. J Neurosci 2010;30:2888-96.

6. Hatton J, et al. Dosing and safety of cyclosporine in patients with severe brain injury. J Neurosurg 2008;109:699-707.

7. Neurovive Pharmaceutical AB. Project overview: cyclophilin-D-inhibiting cyclosporine-based drugs. Available at http://www.neurovive.com/Research-Development/Research-overview. Accessed 10 February 2013.

doi:10.5455/2319-2003.ijbcp20130622

Cite this article as: Singh A, Khare V. Cyclosporin and mitochondria: a neuroprotective approach. Int $\mathbf{J}$ Basic Clin Pharmacol 2013;2:339-40. 